\title{
Bericht vom 26. Bernburger Winterseminar Arznei- und Gewürzpflanzen
}

Am 23. und 24. Februar 2016 fand in Bernburg/ Sachsen-Anhalt mit mehr als 240 Teilnehmern das diesjährige Winterseminar der Saluplanta e.V. statt, in dessen Mittelpunkt wiederum aktuelle Themen aus den verschiedenen Teilbereichen der Arznei- und Gewürzpflanzenproduktion bis hin zur Anwendung der Produkte standen. Doz. h.c. Dipl.-Ing. Bernd Hoppe und Dr. Wolfram Junghanns führten als Gastgeber durch das Programm.

\section{Dauerbrenner Health Claims}

Phytopharmaka im gesundheitspolitischen und regulatorischen Umfeld beleuchtete Dr. Nicole Armbrüster, Berlin. Von Ausnahmen abgesehen, seien Phytopharmaka nicht erstattungsfähig und im Bereich der gesamten Komplementärmedizin gebe es kaum Forschungsförderung, alle Professuren in diesem Bereich seien über Stiftungen finanziert. Neben der aktuellen Arbeit des Herbal Medicinal Products Committee (HMPC) an Monografien und Leitlinien richtet sich der Blick auf Nahrungsergänzungsmittel als „angrenzende" Produktgruppe und die bislang nicht erfolgte Bewertung der gesundheitsbezogenen Aussagen („Health Claims“) von deren pflanzlichen Bestandteilen, den sog. „Botanicals“. Da solche nicht geprüften Claims nicht verboten seien, würden sich hierdurch Wettbewerbsnachteile für pflanzliche Arzneimittel ergeben. Der mit Spannung erwartete „Fitness-Check“ des europäischen Rechtsrahmens zur Bewertung dieser Claims bleibe deshalb abzuwarten.

Dass die Phytotherapie in bestimmten Fällen eine chemisch-synthetische Standardtherapie ersetzen kann, beschrieb

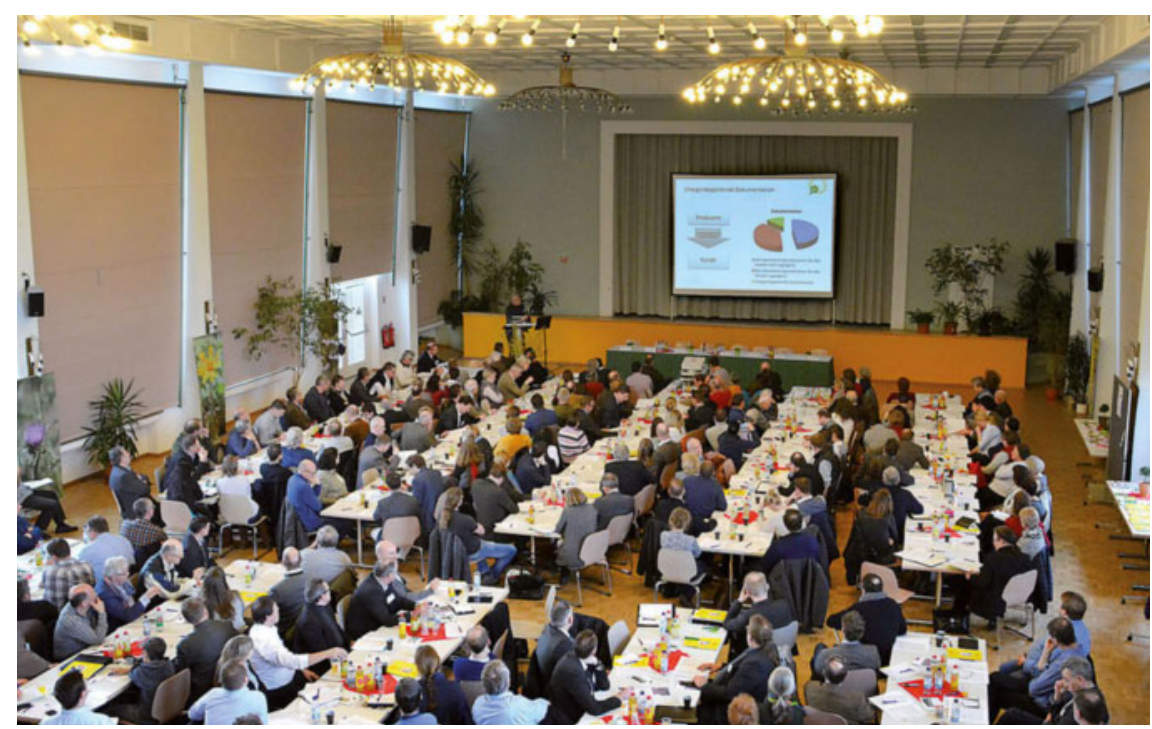

Blick in den Veranstaltungssaal beim Bernburger Winterseminar. (c) Saluplanta e.V.

Dr. Olaf Kelber, Darmstadt, an den Beispielen von funktionellen Magen-DarmErkrankungen, bei denen sich der hohe Stellenwert definierter Kombinationen gezeigt habe, sowie Depressionen, bei denen die Wirksamkeit bestimmter Johanniskrautextrakte klinisch belegt sei. Bei Reizhusten konnte für Eibischextrakt ein schneller Wirkungseintritt gezeigt werden. In den S3-Leitlinien zur Pneumologie beispielsweise seien pflanzliche Arzneimittel berücksichtigt.

\section{Analytik braucht geprüfte Methoden}

Die Erarbeitung spezifischer Gehaltsbestimmungsmethoden wie HPLC oder GC in den Monografien des Europäischen Arzneibuchs erläuterte Dr. Bernhard Klier, Vestenbergsgreuth, Nach Finalisierung der Methodenänderungen bei Orthosiphon und Agnus castus-Früchten seien derzeit die Monografien zu Rosenwurz, Weißdorn, Passionsblume sowie zu den Anthrachinondrogen (insbesondere Sennesblätter und -früchte und Rhabarberwurzel) in Überarbeitung. Der Übergang von unspezifischen (z.B. Photometrie) zu spezifischeren Methoden stelle v.a. bei standardisierten Extrakten eine große Herausforderung dar, da dieser einen direkten Einfluss auf die Deklaration des pflanzlichen Wirkstoffes auf der Packung habe.

Prof. Dr. Johannes Novak, Wien, präsentierte ein von der europäischen Arzneipflanzenanbauer-Vereinigung Europam entwickeltes einheitliches Format für ein Chargenzertifikat pflanzlicher Ausgangsstoffe. Dieses enthält Informationen über das Produkt, die eine Rückverfolgbarkeit erlauben und die Einhaltung der einschlägigen Regelungen (Good Agricultural and Collection Practice, GACP) dokumentieren. Unter dem Motto „das wahre Leben findet auf dem Acker statt“ berich- 


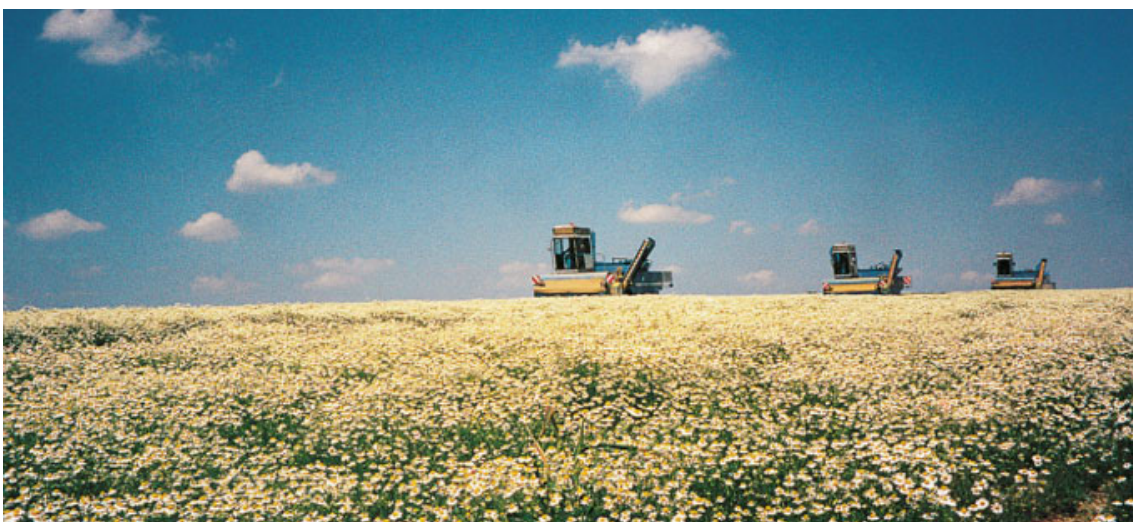

Kamilleernte: Selbst wenige Beikräuter können zu nennenswerten PA-Gehalten führen. Eine Unkrautdatenbank ist im Aufbau. (c) Archiv

tete Dr. Gero Beckmann, Bad Kissingen, über aktuelle Bewertungskriterien von Mikrobiologie und Hygiene bei Arzneiund Gewürzpflanzen und aufgetretene Probleme von Kontaminationen mit Keimen im Lebensmittelbereich sowie die Bedeutung der Prävention bei Produktionsprozessen.

\section{Konzepte zur PA-Problematik}

Dr. Barbara Steinhoff, Bonn, gab einen Überblick über die rechtlichen Bestimmungen zur Prüfung auf Kontaminanten (Pflanzenschutzmittel, Schwermetalle, Mykotoxine) im Europäischen Arzneibuch und angrenzenden Rechtsgebieten des Lebensmittelbereiches. Sie beschrieb die aktuelle Bewertung potenzieller Kontaminationen von pflanzlichem Material mit Pyrrolizidinalkaloiden (PA), für die die arzneipflanzenverarbeitende Industrie in Zusammenarbeit mit den Anbauern eigenverantwortliche Maßnahmen im Sinne eines Code of Practice erarbeitet hat. Mit dieser Empfehlung über die gesamte Prozesskette werde angestrebt, PAKontaminationen langfristig zu reduzieren und die Einhaltung des vom HMPC angesetzten Grenzwertes von 0,35 $\mu$ gA pro Tag im Fertigarzneimittel mittel- bis langfristig zu erreichen. Die Zusammenarbeit aller beteiligten Kreise wie Anbauer und Arzneimittelhersteller, Behörden und Forschungseinrichtungen sei hierbei unabdingbar.

Dr. Andreas Plescher, Artern, stellte Konzept und erste Ergebnisse des von der Fachagentur Nachwachsende Rohstoffe
e.V. (FNR) geförderten Forschungsprojektes zur Erstellung einer Unkrautdatenbank vor, die insbesondere PA-haltige Unkräuter berücksichtigt. Auf ausgewählten Anbauflächen in Deutschland seien in Kulturen von Kamille, Melisse, Pfefferminze und Petersilie bei einem Unkrautaufkommen von ca. 100000 Pflanzen pro Hektar insgesamt 139 Arten identifiziert worden, darunter 6 PA-haltige Arten. Das Kreuzkraut liege dabei auf Rang 43, berücksichtigt werden müsse dabei jedoch, dass selbst wenige Pflanzen dieser Gattung zu nennenswerten PA-Gehalten im Erntegut führen können. Erste Ergebnisse ließen weiterhin darauf schließen, dass die Profile der Unkrautflora in den verschiedenen Kulturen unterschiedlich sind und dass im Kamillenanbau gravierende Bekämpfungslücken bestehen. Das Forschungsprojekt insgesamt stelle deshalb auch ein wertvolles Instrument zur Formulierung von Zielstellungen für Regulierungsmaßnahmen dar.

In diesem Zusammenhang ist auch der chemische Pflanzenschutz bedeutsam, der Thema des Vortrages von Dr. Annette Kusterer, Bernburg war. Sie stellte die Aktivitäten der Landesanstalt für Landwirtschaft und Gartenbau Sachsen-Anhalt dar und gab einen Überblick über die laufenden Untersuchungen und den Stand der Zulassung von Pflanzenschutzmitteln für Arznei- und Gewürzpflanzen, insbesondere welche Mittel für welche Kulturen zur Verfügung stehen.

\section{Krebserkrankungen}

Die Frage, ob gegen Krebs ein Kraut gewachsen ist, untersuchte Prof. Dr. Michael Keusgen, Marburg. Für viele Stoffgruppen wie Polyphenole oder Schwefelverbindungen gebe es Hinweise auf eine tumorpräventive Wirkung, während zur Therapie eher isolierte Reinstoffe wie Alkaloide oder zyklische Peptide, auch in partialsynthetischer Veränderung, zur Anwendung kämen. Allerdings würden viele pflanzliche Therapieansätze nicht weiterentwickelt, weil dies zu aufwändig oder zu teuer sei. Zytostatische Substanzen kämen zahlreich z.B. in tropischen Gewächsen oder marinen Organismen vor, würden aber selten zu pharmazeutischen Präparaten weiterentwickelt, da die unspezifischen zytotoxischen Effekte überwögen.

Als Beispiele für interessante Inhaltstoffgruppen nannte er Catechinderivate im Grüntee, Chalcone im Rooibostee sowie Knoblauch-ähnliche Schwefelverbindungen in Arten der Kaplilie Tulbaghia. Inhaltsstoffe verschiedener Allium-Arten hätten Wirkung auf Blasenkrebszellen gezeigt, allerdings sei die Bioverfügbarkeit fraglich. Relativ gut sei die Datenlage bei der Schlafbeere Withania somnifera, deren überirdische Teile traditionell in der ayurvedischen Medizin verwendet

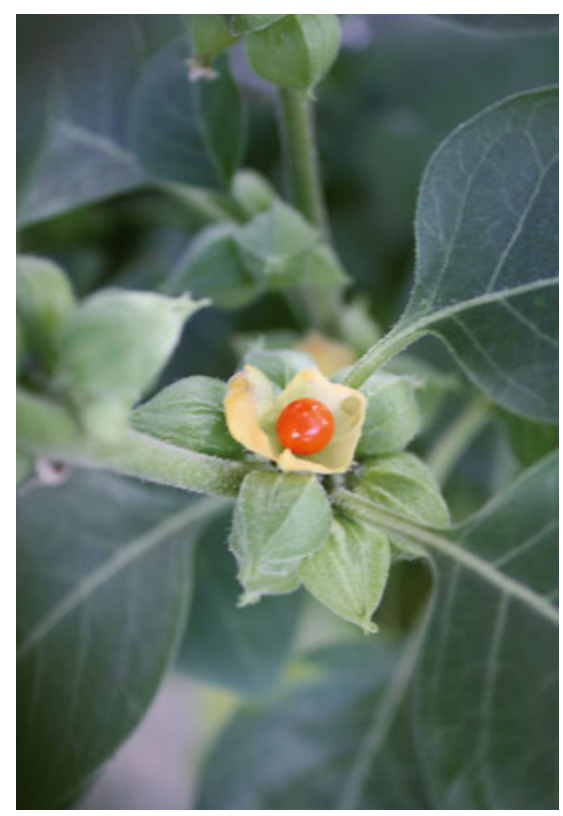

Interessante Datenlage bei Tumorerkrankungen: Withania somnifera (L.) Dunal. (c) Wowbobwow12 
würden. Die Ballonerbse Sutherlandia frutescens enthalte Sterole und den Antimetaboliten Canavanin als Antagonist zur Arginin. Zukunftsweisend könnten auch neue Erkenntnisse zu Cannabis sein, zum einen aufgrund der analgetischen Wirkkomponente Delta 9-THC, zum anderen habe Hanföl Aktivität gegen Tumorzellen gezeigt. Ferner würden Extrakte aus Faserhanf zunehmend klinisch genutzt, was auch für Züchtungsprojekte interessant sein könne. Der Referent warnte allerdings vor Wundermeldungen und irreführenden Laienpresseberichten wie beispielsweise über den Chinesischen Raupenpilz Cordyceps sinensis.

\section{Neue Indikation für Schwarz- kümmel?}

Ein eigener Vortag war der Anwendung von Schwarzkümmel, Nigella sativa, ge- widmet, der traditionell in Asien bei vielfältigen Beschwerden genutzt wird und in Europa als Nahrungsergänzungsmittel erhältlich ist, wie Prof. Dr. Axel Brattström, Magdeburg, darstellte. Einige Studien hätten positive Einflüsse von Schwarzkümmel bzw. dessen Inhaltsstoff Thymochinon bei Stoffwechselerkrankungen gezeigt, weshalb das metabolische Syndrom ein interessantes Einsatzgebiet sein könne (s. ZPT 6/2015).

Weitere Vorträge aus dem Bereich der Züchtung und der Inhaltsstoffforschung befassten sich mit Thymian, Melisse und ätherische Ölen sowie Aufbereitungsprozessen wie der Trocknung von Arzneipflanzen. Über die Tätigkeit des Allrussischen Forschungsinstitutes für Arzneiund Aromapflanzen (VILAR) berichtete Prof. Dr. Elena Malankina, Moskau. Vorträge zum Anbau von Arznei- und Gewürzpflanzen in Chile, in Ungarn und im Iran schlossen sich an.

\section{Ehrungen}

Mit dem diesjährigen Ehrenpreis der Saluplanta e. V. wurde Marut Krusche, Bernburg, für ihre langjährige Tätigkeit im Pflanzenschutz und insbesondere in der Koordination von Versuchen und behördlichen Datensammlungen ausgezeichnet. Den Ehrenpreis der Gemeinnützigen Forschungsvereinigung Saluplanta (GFS) e.V. Bernburg erhielt Dr. Hans Krüger, Quedlinburg, für seine zahlreichen Forschungsaktivitäten am Julius-Kühn-Institut.

\section{Dr. Barbara Steinhoff}

steinhoff@phytotherapie.de

\section{Ein Glas auf das Wohl des Gefäßsystems}

Trinken für die Wissenschaft - das durften Probanden mit leichter Hypercholesterinämie über einen Zeitraum von 10 Wochen. Wie sich das zweimal tägliche Glas Rotwein bzw. Rotwein-Zwiebel-Gemisch auf die Lipidwerte und die antioxidative Kapazität im Blutplasma auswirkte, zeigen die Ergebnisse einer aktuellen klinischen Studie.
Sekundäre Pflanzenstoffe vermitteln vielfältige gesundheitliche Wirkungen. Insbesondere die Polyphenole und Flavonoide haben antioxidatives Potenzial und können so den Organismus vor zahlreichen schädigenden Stoffwechselprodukten schützen. Rotwein steht aufgrund seines hohen Polyphenolgehalts (u.a. Resveratrol) schon länger im Blickpunkt des wissenschaftlichen Interesses rund um Herz-Kreislauf-Erkrankungen und Kardioprotektion. Mit hohen Gehalten an antioxidativ wirkenden Flavonoiden und Schwefelverbindungen gehören auch Zwiebeln zu den Nahrungspflanzen mit gesundheitlichem Mehrwert. 


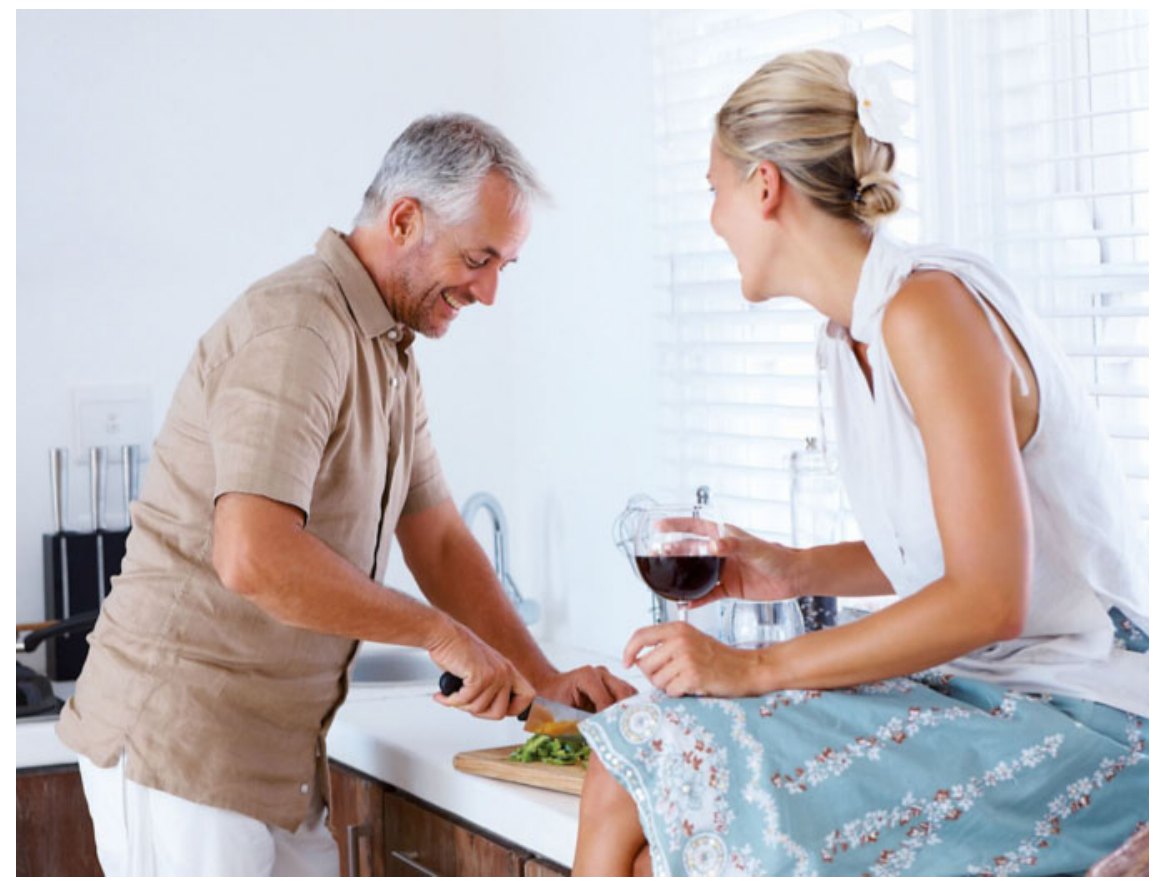

Hält Leib und Seele zusammen: Wein, am besten mit Zwiebeln kombiniert. @ Fotolia/Yuri Arcurs

\section{„Zwiebelwein“ als kombiniert eingesetztes Präventivum}

Dass man den präventiven Nutzen der Inhaltsstoffe von Rotwein und Zwiebeln kombiniert untersuchen könnte, war die Idee einer Forschergruppe aus Taiwan. Sie ließen frisch geschnittene Zwiebeln für 8 Tage in Rotwein ziehen, pürierten sie anschließend und verwendeten das Filtrat (RZ) in einer klinischen Studie im Vergleich zu reinem Rotwein (RW). Insgesamt 23 hypercholesterämische Probanden (Gesamtcholesterin > $180 \mathrm{mg} / \mathrm{dl}$ ) nahmen über einen Zeitraum von 10 Wochen zweimal täglich zur Mittags- und Abendmahlzeit jeweils $125 \mathrm{ml}$ RW oder RZ zu sich. Vor Beginn der Studie sowie nach 2, 6, 10 und 12 Wochen wurden Lipidparameter, Werte zum oxidativen Status und von antioxidativen Enzymen aus dem Blutplasma bestimmt. Zwei Teilnehmer aus der RZ-Gruppe haben die Teilnahme wegen des Zwiebelgeschmacks vorzeitig beendet.

\section{Effektiver Radikalfänger und Lipidsenker}

Bereits in vitro zeigte sich eine deutlich höhere antioxidative Kapazität der beiden Rotweinzubereitungen (RW und RZ) im Vergleich zu einer Zwiebelzubereitung in reinem Alkohol, was die Autoren der Studie auf die im Rotwein im Gegensatz zum reinen Alkohol enthaltenen Polyphenole zurückführen. Dies spiegelte sich dementsprechend auch in vivo wider: In den Plasmaproben der Probanden nahmen die Werte für die Thiobarbitursäure-reaktiven Substanzen (TBARS) als Maß für die Lipidperoxidation deutlich bis in die Follow-up-Phase ab. Dies deutet auf die Zunahme der Radikalfängereigenschaften infolge des Konsums von RW und RZ. Umgekehrt stiegen die Werte für reduziertes Glutathion, für die Glutathionperoxidase und die Glutathionreduktase entsprechend einer gesteigerten antioxidativen Kapazität.

Das Lipidprofil der Probanden entwickelte sich positiv in dem Sinne, dass besonders in der RZ-Gruppe das Gesamtcholesterin und die LDL-Cholesterinwerte deutlich abnahmen. Dies hielt sich bis in die Follow-up-Phase. Hingegen waren die Veränderungen bei den Triglyzeriden und dem HDL-Cholesterin nicht statistisch relevant.

Oxidiertes LDL-Cholesterin kann durch toxische Folgeprodukte das Gefäßendothel schädigen. Letztlich bilden sich dann atherosklerotische Plaques als direkte Faktoren einer kardiovaskulären Erkrankung. Die Einnahme von RZ oder RW führte signifikant zu einer zeitlichen Verzögerung der LDL-Oxidation (gemessen an der Bildung konjugierter Diene). Auch hier war der Effekt wie bei den Lipidwerten in der RZ-Gruppe markanter ausgeprägt - ein Hinweis auf die additive Wirkung der Zwiebelinhaltsstoffe über den Effekt der Polyphenole des Weins hinaus. Dies galt auch für die untersuchten Entzündungsparameter Fibrinogen und Gerinnungsfaktor VII als kardiovaskuläre Risikofaktoren.

\section{Fazit}

Die Einnahme von Rotwein oder einem Zwiebel-Rotwein-Mazerat verbesserte deutlich das antioxidative Potenzial im Blutplasma von hypercholesterämischen Probanden. Der Effekt war unter RZEinnahme ausgeprägter, was auf die additive Wirkung von Polyphenolen, Flavonoiden und organischen Schwefelverbindungen zurückgeführt werden kann. Der kardioprotektive Effekt von RZ scheint demnach ein Zusammenspiel von cholesterinsenkenden, antioxidativen und antiinflammatorischen Eigenschaften zu sein.

Ulrike Andres, Ginsheim

\section{Literatur}

Chiu HF, Shen YC, Huang TY et al. Cardioprotective efficacy of red wine extract of onion in healthy hypercholesterolemic subjects. Phytother Res 2016; 30: $380-385$ 